Article

\title{
Center of Pressure Feedback Modulates the Entrainment of Voluntary Sway to the Motion of a Visual Target
}

\author{
Haralampos Sotirakis ${ }^{1}$, Vassilia Hatzitaki ${ }^{1, *(\mathbb{D}}$, Victor Munoz-Martel ${ }^{2,3}{ }^{\circledR}$, Lida Mademli ${ }^{4}(\mathbb{D}$ and \\ Adamantios Arampatzis ${ }^{2,3}$ \\ 1 Laboratory of Motor Behavior and Adapted Physical Activity, School of Physical Education and Sport \\ Sciences, Aristotle University of Thessaloniki, 54024 Thessaloniki, Greece; sotiraki@phed.auth.gr \\ 2 Department of Training and Movement Sciences, Humboldt-Universität zu Berlin, 10115 Berlin, Germany; \\ v.munozmartel@hu-berlin.de (V.M.-M.); a.arampatzis@hu-berlin.de (A.A.) \\ 3 Berlin School of Movement Science, Humboldt-Universität zu Berlin, 10115 Berlin, Germany \\ 4 Department of Physical Education and Sports Science at Serres, Aristotle University of Thessaloniki, \\ 54024 Thessaloniki, Greece; lmademli@phed-sr.auth.gr \\ * Correspondence: vaso1@phed.auth.gr
}

Received: 13 June 2019; Accepted: 17 September 2019; Published: 20 September 2019

\begin{abstract}
Visually guided weight shifting is widely employed in balance rehabilitation, but the underlying visuo-motor integration process leading to balance improvement is still unclear. In this study, we investigated the role of center of pressure $(\mathrm{CoP})$ feedback on the entrainment of active voluntary sway to a moving visual target and on sway's dynamic stability as a function of target predictability. Fifteen young and healthy adult volunteers (height $175 \pm 7 \mathrm{~cm}$, body mass $69 \pm 12 \mathrm{~kg}$, age $32 \pm 5$ years) tracked a vertically moving visual target by shifting their body weight antero-posteriorly under two target motion and feedback conditions, namely, predictable and less predictable target motion, with or without visual CoP feedback. Results revealed lower coherence, less gain, and longer phase lag when tracking the less predictable compared to the predictable target motion. Feedback did not affect CoP-target coherence, but feedback removal resulted in greater target overshooting and a shorter phase lag when tracking the less predictable target. These adaptations did not affect the dynamic stability of voluntary sway. It was concluded that $\mathrm{CoP}$ feedback improves spatial perception at the cost of time delays, particularly when tracking a less predictable moving target.
\end{abstract}

Keywords: posture; balance; weight shifting; target predictability; coherence; dynamic stability

\section{Introduction}

An integral component of balance rehabilitation exercises is real time visual feedback regarding the instantaneous center of pressure $(\mathrm{CoP})$ or center of mass $(\mathrm{CoM})$ trajectory in relation to a stationary or moving visual target position. Although this visualization increases the role of visual information in the multisensory integration process of controlling posture, its role in standing and dynamic balance control is not well understood.

Experimental evidence on the role of visual feedback in controlling standing balance when the goal of the task is to minimize sway around a reference position is conflicting. On one hand, real time CoP or CoM feedback decreased sway amplitude [1] and the instability induced by proprioceptive, somatosensory, and vestibular perturbations [2]. On the other hand, augmented feedback of the two-dimensional CoP or CoM position did not improve postural stability of a two-legged quiet standing posture [3], whereas, when standing participants were exposed to a complex visual room oscillation, provision of CoP feedback further destabilized standing sway [4]. Furthermore, the spatial 
CoP variability of visually controlled whole-body leaning did not decrease when performance feedback was provided in addition to only target information [5]. A possible explanation could be the fact that visual feedback operates at a relatively low frequency scale and therefore it is not appropriate for correcting the high-frequency, small-amplitude CoP oscillations of standing sway [6]. On the other hand, feedback may have a more prominent impact on dynamic equilibrium control which involves greater, longer, and lower frequency $\mathrm{CoP}$ fluctuations (drifts) over the base of support.

Provision of augmented visual feedback during performance of dynamic balancing tasks revealed more systematic effects. Removing visual CoP feedback during lateral weight shifting decreased the speed and spatial accuracy of performance [7]. However, the same removal did not have an impact on performance when participants swayed voluntary while trying to produce different visually imposed ankle-hip coordination patterns represented in a complex Lissajous figure [8]. This suggests that more simple and direct forms of visual feedback are needed in order to improve performance. Indeed, the provision of two-dimensional instead of one-dimensional information resulted in faster lateral weight-shifting [9], while augmentation of the error feedback signal drove subjects to their steady-state performance faster than unaltered visual feedback [10]. Horizontally biased visual feedback induced horizontal compensatory postural adjustments, which increased CoP asymmetry even in quiet standing after practice [11]. This evidence suggests that augmented, real-time visual feedback regarding sway improves spatial accuracy and speed of performance when the goal of the task is to transfer the body toward a stationary visual target, such as in lateral weight shifting.

The role of visual feedback when actively tracking a moving visual target with the whole body is less studied. Work from our laboratory suggests that humans, regardless of their age, can couple their voluntary sway to a moving visual target regardless of whether this oscillates in a stereotypical (i.e., periodically) or in a more complex (i.e., chaotically) fashion [12]. However, the role of CoP feedback in visuo-postural entrainment and its dependence on target predictability is still not known. The extent to which the body's stability is challenged by the availability of feedback and target predictability during active target tracking is another unresolved issue. Local dynamic stability represents the ability of a system to maintain its movement pattern despite intrinsic and extrinsic perturbations $[13,14]$. Instantaneous visual feedback of the pelvis and trunk motion in the frontal plane during gait reduced the local dynamic stability of the aforementioned segmental movements, suggesting that visual feedback of the segment motions induces additional frontal plane instability during gait [15]. The reasons for this increase are still not well understood.

In order to address the above challenges, we examined the role of visual feedback on visuo-postural coupling and dynamic stability of sway when actively tracking either predictable (periodic) or less predictable (chaotic) target motion cues in the sagittal plane. We hypothesized that when actively tracking a visual target oscillating vertically with the body, the impact of feedback would depend on the predictability of the visual motion cues. Specifically, two predictions were tested: (a) That the removal of feedback would affect the strength of visuo-motor coupling and the dynamic stability of sway, and (b) that the impact of feedback would be greater when tracking the less predictable target motion.

\section{Materials and Methods}

\subsection{Participants}

Fifteen healthy adults ( 10 males, 5 females, height $175 \pm 7 \mathrm{~cm}$, body mass $69 \pm 12 \mathrm{~kg}$, age $32 \pm 5$ years, mean $\pm \mathrm{SD}$ ), recruited among the university staff participated in this study. None of the participants had a history of neuromuscular impairments or balance-related dysfunctions. No participants used orthotic insoles, and all had normal or corrected-to-normal vision. All participants were informed about the experimental protocol and gave their informed consent prior to their inclusion in the study. The experiment was performed with the approval of the institution's ethics committee (HU-KSBF-EK_2018_0013, Humboldt-Universität zu Berlin) in accordance with the Declaration of Helsinki. 


\subsection{Apparatus, Stimuli, and Task}

Postural performance was recorded using a force platform $(60 \times 90 \mathrm{~cm}$, Kistler, $1000 \mathrm{~Hz}$, Winterthur, Switzerland). Visual stimuli were displayed on a large TV Screen (47 inch, HD LG), located $1.5 \mathrm{~m}$ in front of the participant at eye level (Figure 1A).

The target's motion was constructed in MATLAB (version R2014b, Math Works Inc, Natick, MA, USA) using two signals of different degrees of predictability. The predictable signal was a sinewave with a single frequency (f) set at $0.25 \mathrm{~Hz}$ that was generated using the $\sin$ function $[\operatorname{sine}=\sin (2 p i \times \mathrm{f} \times t)]$. This particular frequency was selected because it was the dominant frequency of intuitive, self-paced voluntary sway based on prior studies $[12,16]$ and pilot testing. The less predictable signal was derived from a Lorenz attractor according to the parameters: $\sigma=10, \beta=8 / 3$, and $r=28$ and the initial conditions: $\mathrm{x} 0=0.1, \mathrm{y} 0=0.1$, and $\mathrm{z} 0=0.1$. The signal characteristics were $\mathrm{h}$ (time resolution) $=0.0040$, steps (number of points) $=10,000$ (we choose 6000 data points from the y-axis [y $(4000: 10,000)$ ], and noise flag $=0[17,18]$. The frequency range for the Lorenz signal was confined between 0 and $1 \mathrm{~Hz}$, which was an ecologically valid spectrum of frequencies for voluntary sway [19], and its power spectrum revealed a dominant frequency around $0.25 \mathrm{~Hz}$.

Prior to the experiment, participants were asked to step on the platform and adopt the testing position with their arms freely hanging by their sides. The distance between the internal malleoli was set at $10 \%$ of body height. The antero-posterior component of the base of support (foot length) was measured using the distance of the most anterior point (toe) to the calcaneus in order to normalize the amplitude of target motion. The maximum (peak to peak) amplitude of target motion was set to $60 \%$ of the foot length (Figure 1B).

During the experiment, participants tracked the motion of the visual target, which moved vertically on the screen, with their bodies by shifting their $\mathrm{CoP}$ in the antero-posterior direction. Two different-colored dots were shown on the screen, specifically, a red dot, which illustrated the target to be followed, and a yellow dot, which depicted the participant's antero-posterior CoP component serving as the instantaneous performance feedback signal. The only instruction given to participants was to follow the motion of the red dot by controlling the motion of the yellow one, which was achieved by shifting their weight antero-posteriorly. The two stimuli (target and feedback) were synchronously represented on the TV monitor using custom build software (developed in MATLAB) while their position was updated at a rate of $50 \mathrm{~Hz}$, providing $120 \mathrm{~s}$ of continuous target stimulus motion and resulting in 6000 data points for each signal. Participants performed one $120 \mathrm{~s}$ long tracking trial in each of the following conditions: (a) Tracking of the sinusoidal target with (yellow dot was visible) and without (yellow dot was not visible) visual feedback, and (b) tracking of the chaotic (Lorenz) target with and without feedback. Task conditions were randomized to avoid possible order effects. Participants were provided ample time to familiarize themselves with the task prior to actual testing. Prior to each trial, the room lights were dimmed. Between each trial, participants rested for about $2 \mathrm{~min}$. The experimental session, including subject preparation lasted no longer than $60 \mathrm{~min}$. 
A

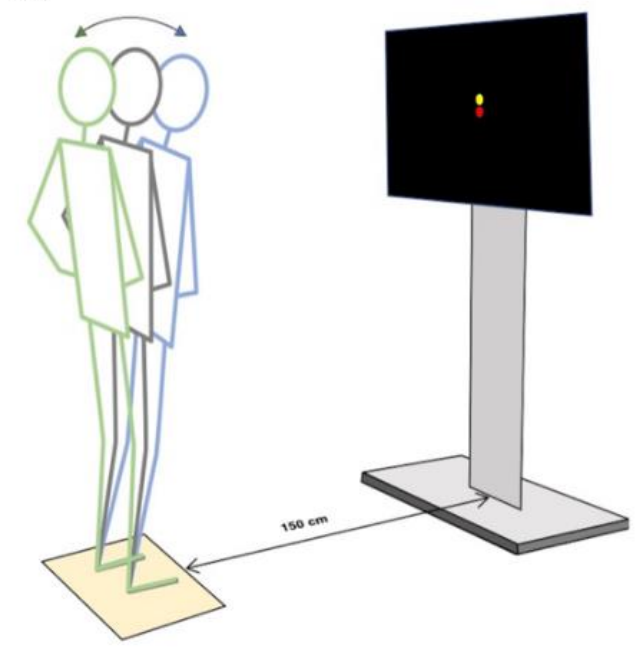

B

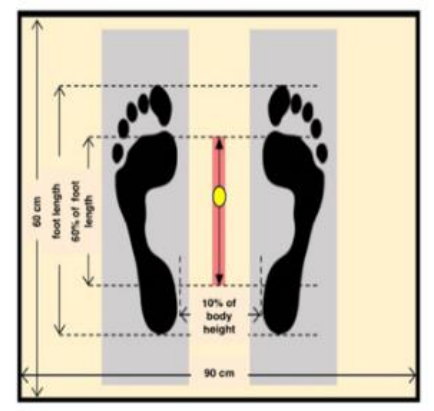

Figure 1. Graphical illustration of the apparatus and task. (A) Participants were asked to track the visual (red) target dot moving in the vertical direction with their body by shifting their center of pressure (CoP) (yellow dot) in the antero-posterior direction. An upward target motion was tracked by a forward CoP shift, while a downward target motion was tracked by a backward CoP shift. (B) The target's (red dot) motion amplitude was normalized to $60 \%$ of the participants foot length. Tracking lasted $120 \mathrm{~s}$ and was performed either with feedback (yellow dot was visible) or without feedback (yellow dot was not visible).

\subsection{Data Analysis}

Center of pressure (CoP) and target time series were processed and analyzed in MATLAB (R2014b). The original antero-posterior component of the CoP time series was down-sampled to $50 \mathrm{~Hz}$ in order to match the screen update rate of the target signal display prior to low pass filtering using a 4th order Butterworth low-pass filter, with a cutoff frequency at $5 \mathrm{~Hz}$.

\subsection{Spectral Coherence}

The CoP-target motion coupling was assessed using spectral analysis in the frequency band between $0-1 \mathrm{~Hz}$, based on the methods of Halliday et al. [20]. For this purpose, a customized version of a freely available software (NeuroSpec 2.0) was used. Both the CoP and target time series were interpolated at $64 \mathrm{~Hz}$ in order to achieve an appropriate frequency resolution for the spectral analysis, as we wanted to get a coherence, phase, and gain value of $0.25 \mathrm{~Hz}$ (frequency of the sinewave) and the neighborhood frequencies (for the Lorenz signal). Spectral analysis was performed in the interpolated time series of 7680 data points which were sampled at $64 \mathrm{~Hz}$. Setting the segment length power at $2^{10}$ (1024 data points) gave a segment duration of $16 \mathrm{~s}$. This resulted in a frequency resolution of 0.0625 $\mathrm{Hz}$. The software output the means and confidence limits across the entire frequency band $(0-1 \mathrm{~Hz})$ according to three variables, i.e., spectral coherence, spectral phase, and spectral gain. The spectral coherence was used as a metric of correlation between the two signals (CoP-target position) in the frequency domain, illustrating their linear relationship. The spectral phase illustrated the temporal relationship between the two signals, expressed in degrees $\left({ }^{\circ}\right)$. The absolute synchronization between the two signals was illustrated by $0^{\circ}$ phase lag, while positive and negative values indicated that the sway led or followed the target motion respectively. The spectral gain revealed information regarding the spatial (amplitude) coupling between the two signals (CoP-target), while a gain of 1 indicated an absolute spatial coupling at a given frequency point. Gain values less than or greater than 1 indicated target undershooting or overshooting (the CoP moved with smaller or greater amplitude than the target), respectively. For the sinewave, the coherence, phase, and gain values at the target's dominant frequency $(0.25 \mathrm{~Hz})$ were computed and registered for statistical analysis. Similarly, for the Lorenz 
target signal, the average of the coherence, phase, and gain values at 3 adjacent frequency bins $(0.1875$, 0.25 , and $0.3125 \mathrm{~Hz}$ ) was calculated.

\subsection{Local Dynamic Stability}

The local dynamic stability of the system in the current study was assessed using the maximum finite-time Lyapunov exponent (MLE), which quantifies the rate of divergence of nearby trajectories in state space [21,22]. The analysis followed the procedure used in a previous study [23]. The MLE was calculated on the norm of the anterio-posterior and mediolateral CoP. Initially, the original time-series were filtered using a 4th order Butterworth low-pass filter with a cut-off frequency of $20 \mathrm{~Hz}$ and consequently down-sampled to 20,000 data points. Due to the standardized overall trial duration (i.e., $120 \mathrm{~s}$ ), no interpolation of the time-series was needed. To reconstruct the state space from the one-dimensional time series, we used delay-coordinate embedding [24] as follows:

$$
S(t)=[z(t), z(t+\tau), \ldots, z(t+(m-1) \tau)]
$$

where $S(t)$ is the m-dimensional reconstructed state vector, $z(t)$ is the input $1 \mathrm{D}$ coordinate series, $\tau$ is the time delay, and $m$ is the embedding dimension. Time delays were selected based on the first minimum of the Average Mutual Information function [25]. For these data, $m=3$ was sufficient to perform the reconstruction. Individually selected time delays were chosen by averaging the outcome delays deriving from both trials performed by the participants [26], with $\tau$ ranging from $\sim 0.20$ to $\sim 0.27$ of the overall cycle. Further, the average divergence of each point's trajectory to its closest neighbor was calculated using the Rosenstein algorithm [27]. The resulting MLE was calculated based on the delay of each participant, which ensured the standardization of the calculation for the MLE across all of the individuals. Commonly, the first peak in the resulting divergence curves corresponded to a delay of 0.5 (percentage of the average postural sway cycle). The final MLE value was calculated as the slope of the average divergence curves' linear fit which corresponded to the individuals' delay values at 0.25 of the average postural sway cycle (i.e., the most linear part of the curve).

\subsection{Statistical Analysis}

Prior to statistical analysis, the Shapiro-Wilk test was applied to test for violations of the normality assumption in each outcome measure. All measures were normally distributed and differences between the two target motions (periodic and chaotic) and feedback conditions (with and without visual feedback) on the CoP-target coherence, phase, and gain were compared by employing a 2 (target) $\times 2$ (feedback) ANOVA model with repeated measures on both factors. Target by feedback interactions were further analyzed using pairwise comparisons between the feedback conditions, which were performed separately on each target motion. Differences in the resulting MLE values were examined using Student's $t$-test via pairwise comparisons between the two feedback conditions. Effect sizes were reported using $\mathrm{h}^{2}$. Statistical analysis was performed using SPSS (v.24).

\section{Results}

Group-averaged CoP displacement and target signals are shown in Figure 2 for sinusoidal and chaotic target tracking, respectively, with and without feedback. 

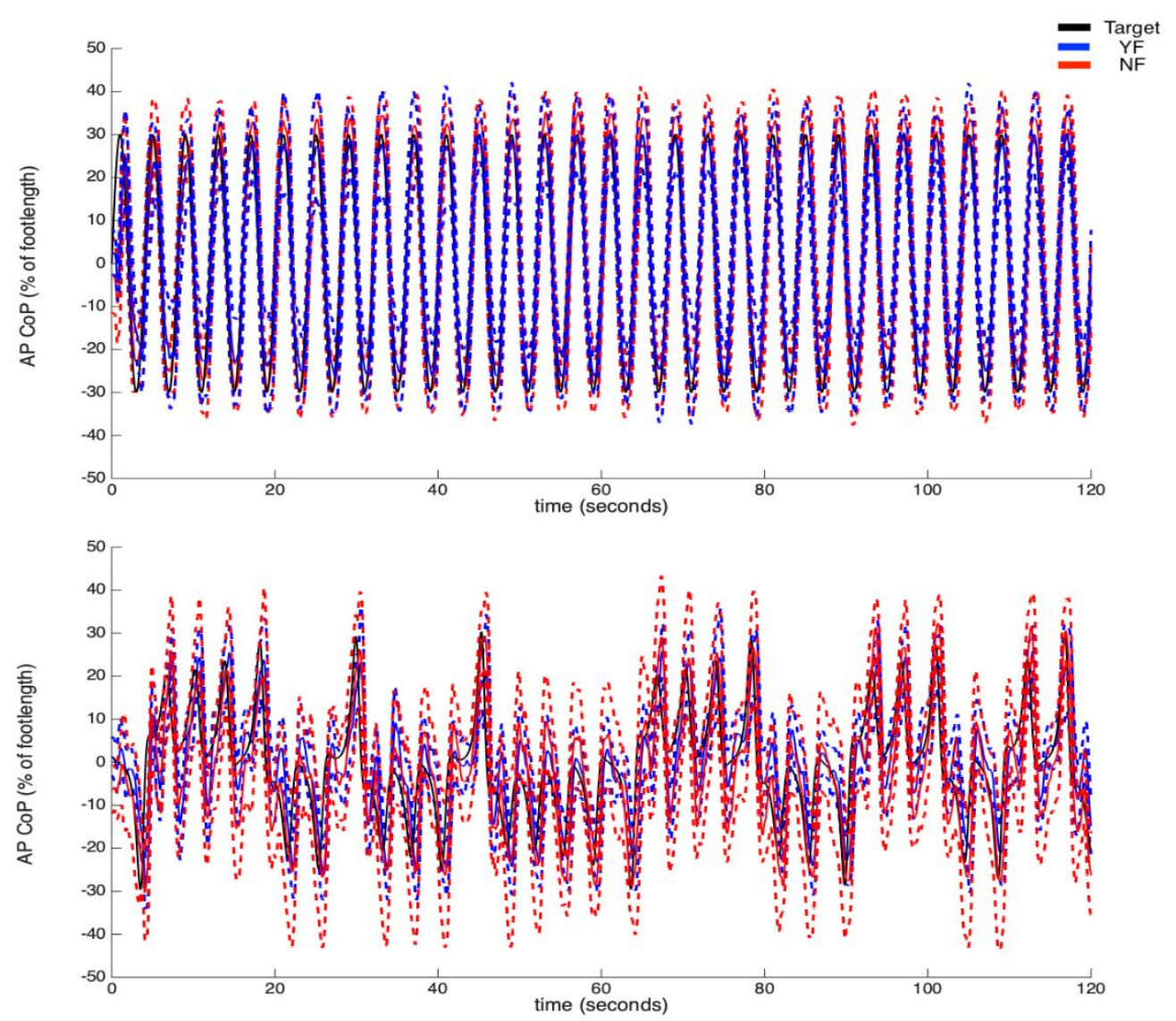

Figure 2. Group-averaged ( $n=15) \mathrm{CoP}$ and target (black) time series during performance of (1st row) the sinusoidal and (2nd row) the chaotic tracking with (blue) and without (red) feedback. Dotted lines indicate the group $95 \%$ confidence intervals.

\subsection{Spectral Analysis}

\section{- $\quad$ Coherence}

The CoP-target coherence values ranged between 0.75 and 0.99 for all participants under all tracking conditions (Figure $3 \mathrm{~A}$ ), suggesting that all participants were successful in tracking the target. However, CoP-target coherence decreased significantly with chaotic tracking relative to the periodic target $\left(\mathrm{F}(1,14)=264, p=0.000, \mathrm{~h}^{2}=0.950\right)$, although this was not different between the two feedback conditions.

- $\quad$ Phase

The CoP-target phase lag significantly increased when tracking the chaotic compared to the periodic target $\left(\mathrm{F}(1,14)=67.19, p=0.000, \mathrm{~h}^{2}=0.828\right)$. A negative phase lag value indicated that the $\mathrm{CoP}$ followed the target motion (Figure $3 \mathrm{~B}$ ). A significant feedback by target interaction effect on the phase lag $\left(\mathrm{F}(1,14)=8.51, p=0.011, \mathrm{~h}^{2}=0.378\right)$ suggested that the effect of feedback was dependent on the target motion. Post hoc analysis confirmed that the presence of visual feedback significantly increased the CoP-target phase lag compared to the no feedback condition only in chaotic target tracking $(\mathrm{t}(16)=2.93, p=0.010)$. On the other hand, the feedback signal had a non-significant effect on the CoP-target phase lag when tracking the periodic target motion.

\section{- Gain}

Removal of feedback resulted in a significant increase in the CoP-target gain $(\mathrm{F}(1,14)=68.49$, $\left.p=0.000, h^{2}=0.830\right)$, suggesting that target overshooting occurred during both periodic and chaotic 
target tracking (Figure 3C). More target overshooting was observed when tracking the chaotic target compared to the periodic target $\left(\mathrm{F}(1,14)=77.57, p=0.000, \mathrm{~h}^{2}=0.847\right)$. The impact of visual feedback on CoP-target gain was significantly greater when tracking the chaotic compared to the periodic target, as confirmed by a significant feedback by target interaction effect $\left(\mathrm{F}(1,14)=22.16, p=0.000, \mathrm{~h}^{2}=0.613\right)$. Specifically, the increase in CoP-target gain induced by the removal of feedback was greater when tracking the chaotic compared to the sinusoidal target $(\mathrm{t}(16)=7.81, p=0.000)$.
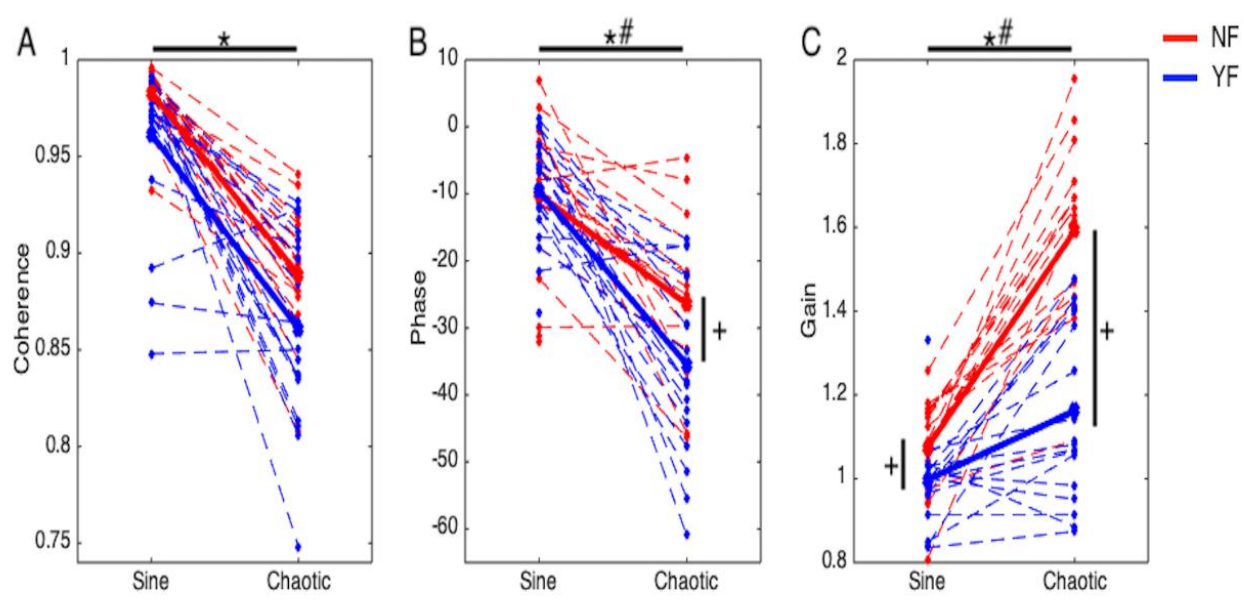

Figure 3. Center of pressure $(\mathrm{CoP})$-target: $(\mathbf{A})$ coherence, $(\mathbf{B})$ phase, and $(\mathbf{C})$ gain during tracking of the sinusoidal and chaotic target motion with (YF, blue) and without (NF, red) CoP feedback. Dashed lines indicate individual cases and group means are shown with solid bold lines. +: Significant difference between feedback conditions at $p<0.05$; *: Significant difference between target conditions at $p<0.05$; \#: Significant feedback by target interaction effect at $p<0.05$.

\subsection{Local Dynamic Stability}

The group means and standard deviations for the MLE exponent are shown in Figure 4. The dynamic stability index of the normalized CoP displacement was not affected by the presence of feedback when tracking either the sinusoidal or the chaotic target. This was confirmed by the absence of a significant difference between the feedback conditions in both the periodic $(p=0.463)$ and chaotic $(p=0.276)$ target tracking.

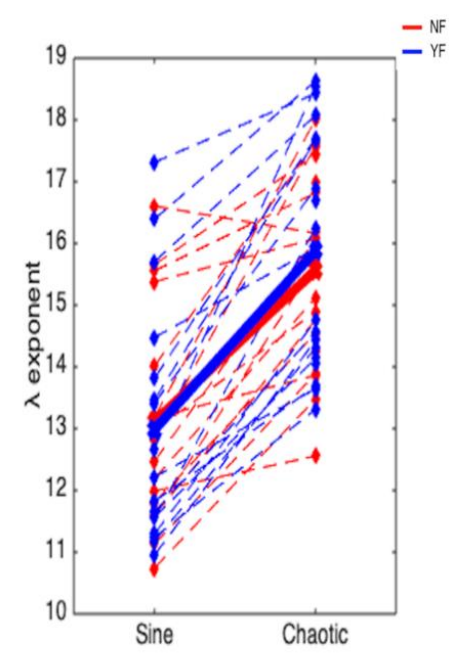

Figure 4. Maximum Lyapunov exponent (MLE) values of the CoP time series during tracking of the sinusoidal target motion and chaotic target motion with (YF, blue) and without (NF, red) CoP feedback. Dashed lines indicate individual cases and group means are shown with solid bold lines. 


\section{Discussion}

In accordance with our hypotheses, the present results confirmed an effect of real-time CoP feedback on visuo-motor coupling during active postural tracking of a vertically moving visual target. The effect was stronger when tracking the more variable (i.e., chaotic) target motion. On the other hand, the presence/removal of feedback did not affect the dynamic stability of voluntary sway.

- Feedback improved spatial accuracy at the cost of time delays.

When real-time visual feedback of the CoP trajectory relative to the target motion was available during active target tracking, the CoP-target gain was close to one. This suggests that sway amplitude closely matched the target oscillation amplitude that was set to the individual stability boundaries in the antero-posterior direction (60\% of foot length). We argue that real-time feedback regarding the instantaneous $\mathrm{CoP}$ position provided critical spatial information for actively controlling sway amplitude through an error correction process, which allowed performers to make corrective postural adjustments before horizontal $\mathrm{CoP}$ accelerations led to large deviations in postural sway. On the other hand, removing $\mathrm{CoP}$ feedback during tracking of the vertical target motion increased the CoP-target gain, suggesting target overshooting. Our results are concordant with those of a previous study showing that augmented CoP feedback improved the spatial accuracy of lateral weight shifting [7]. However, instead of translating the body toward a stationary target, in our protocol, the task goal required active and dynamic tracking of the moving target. Target overshooting in the absence of feedback in this case implies larger antero-posterior CoP acceleration and, consequently, increased difficulty and greater muscular effort in controlling the reversal in sway direction [28,29]. In this case, the control of active sway relied on an internal representation of the body schema in space, which tended to overestimate the sensory consequences of the actual tracking task relative to the boundaries of the target's motion, resulting in erroneously larger sway movements [30]. Internal representations of the body schema are based on prior knowledge and appear to be central in the planning and control of goal-directed actions [31,32]. The absence of error information in this case is related with exploratory behavior of the spatial task constraints and over-corrections [33,34]. Greater reliance on prediction is also associated with greater perceptual bias [30], which may explain the overestimation of the target boundaries and the target overshooting observed in the absence of feedback.

On the other hand, availability of feedback increased the phase lag between the target and the CoP motion when tracking the chaotic target, resulting in slower antero-posterior weight shifting. This finding contradicts previous studies showing that provision of visual feedback resulted in faster lateral weight shifting $[10,35]$. The discrepancy could be due to the stationary nature of the target used in the previous weight shifting paradigms as opposed to the moving target used in the present study. When the target was stationary, information about the target's position was picked up prior to the onset of movement, which allowed for faster lateral weight transfer. However, in the case of a moving target, the target's position was continuously updated during task performance, while real-time feedback about the instantaneous CoP position imposed a greater need for online visuo-motor integration, resulting in time delays when making the appropriate postural adjustments. In this case, feedback may have acted as additional sensory noise that increased the time delay (phase lag) between the target and sway motion [36]. On the other hand, periodic target tracking did not increase the phase lag between postural sway and the target motion due to the predictable nature of the target motion. Once a particular visuo-motor transformation was learned after a couple of sway cycles, the participant was able to predict the target's motion, thereby reducing the load of online visual information processing during active target tracking [16].

Overall, our results suggest a trade-off in the control of the spatial and temporal properties of sway when tracking a vertically moving target which was modulated by the availability of visual CoP feedback. Feedback about the spatial error between the target and the CoP motion induced a prioritization of spatial over temporal coupling [37], while the latter decreased due to the speed accuracy trade-off [38]. 
- Feedback did not affect the dynamic stability of voluntary sway.

Removal of feedback did not affect the local dynamic stability of sway. This suggests that the additional visuo-motor processing load needed for matching the feedback (yellow dot) to the target signal (red dot) in the feedback condition did not challenge the overall dynamic stability of the voluntary sway task any more than the target tracking alone with no feedback. Along the same line of evidence, in an optic flow stimulation study, participants experienced less postural instability during the actual saccadic tracking of the directional stimuli compared to the preceding perceptual phase of the heading direction [39]. This suggests that when the visual motion of the target was congruent with the eye and/or body motion, the postural stability was not challenged. On the other hand, provision of real-time visual feedback during gait decreased the local dynamic stability and induced instability of the trunk and the pelvis in the frontal plane [15]. Several reasons could explain this disagreement. Actively tracking a moving target by shifting the body weight in the sagittal plane is a less automatic and slower task compared to gait. Provision of augmented feedback during gait could therefore lead to a disruption of automaticity and impose greater demand for visuo-motor processing. Weight-shifting, on the other hand, is a less automatized task that requires higher precision control, particularly when reaching close to the stability limits. The plane of motion could also be an important task constraint determining the impact of dynamic visual feedback cues on dynamic stability [40]. In the gait study, feedback was represented by a horizontal visual motion to inform about the instantaneous position of the pelvis and the trunk in the frontal plane, whereas in our paradigm, CoP feedback, provided by a vertically moving dot, did not seem to affect the stability of sway in the sagittal plane. It is known that non-gravity (horizontal)-related dynamic visual cues induce greater instability when compared to gravity-relevant visual cues [40]

- The contribution of feedback increased when tracking the less predictable target.

Tracking of the chaotic target resulted in lower coherence, greater target overshooting, and a longer phase lag between the target and the CoP motion when compared to periodic target tracking. These results confirm previous findings from our laboratory showing that voluntary, sagittal plane sway synchronizes better with predictable (i.e., periodic) target motion than less predictable (i.e., chaotic) target motion when actively tracking a vertically moving target $[12,16]$. It could be suggested that postural tracking of the chaotic target imposed more extensive visuo-motor processing due to the less predictable nature of the target's motion, which may have kept participants more actively engaged in the tracking task due to the continuous need to attend to the target motion. Tracking of the sinusoidal target motion, on the other hand, gradually decreased the need to attend to the target, as participants were able to predict it and adjust their sway, employing an open-loop type of control [32]. This was confirmed by the consistency of the target-CoP phase lag across the two feedback conditions when tracking the sinusoidal target motion. Moreover, synchronizing body sway to a chaotically oscillating target may also involve anticipatory control, which depends on the availability of visual feedback [41]. This process involves short-term prediction and correction of asynchronies between the target and the CoP motion [42]. Instead of prediction on a local time-scale, however, strong anticipation assumes coordination on a longer time-scale. Entraining to a chaotic target motion in this case would require the coordination of posture to the global temporal structure of the stimulus signal and not on the local temporal changes [5].

The greater target overshooting when tracking the less predictable target may also have been due to the different amplitude scaling of the chaotic signal. The chaotic target motion-imposed direction reversed at smaller sway amplitudes, as the target's motion did not consistently reach the stability limit, which was set at $60 \%$ of foot length in every sway cycle. The most important information for stabilizing coordination with an oscillating stimulus was available for the actor around the endpoint of its trajectory [43]. Thus, it may have been more difficult to control smaller oscillation amplitudes during active sway than target amplitudes which reached the stability limit [44]. 


\section{Conclusions}

Feedback may be more critical for spatial coupling when accuracy is a task requirement, while it imposes an extra processing constraint for synchronizing voluntary sway to the moving target stimulus. Models of postural control have highlighted possible roles for both feedback-based [45] and predictive, feed-forward control [46] in the control of voluntary sway during static and dynamic balance activities. Both control processes are compatible with these current behavioral results. The observed changes in postural sway control as a function of feedback availability may reflect an updating of internal models relating postural motor commands to their upcoming sensory consequences (i.e., forward models), facilitating accurate on-line detection and correction of postural deviations (feedback control).

Author Contributions: Conception and design of the work A.A., V.H., and L.M.; data acquisition H.S. and V.M.-M.; data analysis H.S. and V.M.-M.; data interpretation V.H., A.A., and L.M.; first draft H.S.; manuscript revision A.A., V.H., and L.M.; manuscript editing, H.S. and V.M.-M. All authors approved the submitted version to be published and agreed to be accountable for all aspects of the work.

Funding: This research was part of the project "FFP-AGE", which was funded by the German Academic Exchange Service (DAAD); grant number: 57339989.

Acknowledgments: Authors would like to thank Arno Schroll for the creation of the interface for data collection and the hardware setup, Antonis Ekizos for contributing to the data analysis, and Sebastian Bohm and Natasa Papavasileiou for their assistance in data collection and figure preparation.

Conflicts of Interest: The authors declare no conflict of interest.

\section{References}

1. Rougier, P. Visual feedback induces opposite effects on elementary centre of gravity and centre of pressure minus centre of gravity motions in undisturbed upright stance. Clin. Biomech. 2003, 18, 341-349. [CrossRef]

2. Cofré Lizama, L.E.; Pijnappels, M.; Reeves, N.P.; Verschueren, S.M.P.; van Dieën, J.H. Can explicit visual feedback of postural sway efface the effects of sensory manipulations on mediolateral balance performance? J. Neurophysiol. 2016, 115, 907-914. [CrossRef] [PubMed]

3. Kilby, M.C.; Slobounov, S.M.; Newell, K.M. Augmented feedback of COM and COP modulates the regulation of quiet human standing relative to the stability boundary. Gait Posture 2016, 47, 18-23. [CrossRef] [PubMed]

4. Li, R.; Peterson, N.; Walter, H.J.; Rath, R.; Curry, C.; Stoffregen, T.A. Real-time visual feedback about postural activity increases postural instability and visually induced motion sickness. Gait Posture 2018, 65, $251-255$. [CrossRef] [PubMed]

5. Duarte, M.; Zatsiorsky, V.M. Effects of body lean and visual information on the equilibrium maintenance during stance. Exp. Brain Res. 2002, 146, 60-69. [CrossRef] [PubMed]

6. Ivanenko, Y.; Gurfinkel, V.S. Human postural control. Front. Neurosci. 2018, 12, 1-9. [CrossRef]

7. Dault, M.C.; De Haart, M.; Geurts, A.C.H.; Arts, I.M.P.; Nienhuis, B. Effects of visual center of pressure feedback on postural control in young and elderly healthy adults and in stroke patients. Hum. Mov. Sci. 2003, 22, 221-236. [CrossRef]

8. Faugloire, E.; Bardy, B.G.; Merhi, O.; Stoffregen, T.A. Exploring coordination dynamics of the postural system with real-time visual feedback. Neurosci. Lett. 2005, 374, 136-141. [CrossRef] [PubMed]

9. Kennedy, M.W.; Crowell, C.R.; Striegel, A.D.; Villano, M.; Schmiedeler, J.P. Relative efficacy of various strategies for visual feedback in standing balance activities. Exp. Brain Res. 2013, 230, 117-125. [CrossRef]

10. O'Brien, K.; Crowell, C.R.; Schmiedeler, J. Error augmentation feedback for lateral weight shifting. Gait Posture 2017, 54, 178-182. [CrossRef]

11. Shiller, D.M.; Veilleux, L.-N.; Marois, M.; Ballaz, L.; Lemay, M. Sensorimotor adaptation of whole-body postural control. Neuroscience 2017, 356, 217-228. [CrossRef] [PubMed]

12. Sotirakis, H.; Kyvelidou, A.; Stergiou, N.; Hatzitaki, V. Neuroscience Letters Posture and gaze tracking of a vertically moving target reveals age-related constraints in visuo-motor coupling. Neurosci. Lett. 2017, 654, 12-16. [CrossRef] [PubMed]

13. Lyapunov, A.M. The general problem of motion stability. Int. J. Control 1992, 55, 531-773. [CrossRef] 
14. Ihlen, E.A.F.; van Schooten, K.S.; Bruijn, S.M.; Pijnappels, M.; van Dieën, J.H. Fractional stability of trunk acceleration dynamics of daily-life walking: Toward a unified concept of gait stability. Front. Physiol. 2017, 8, 516. [CrossRef] [PubMed]

15. Hamacher, D.; Hamacher, D.; Schega, L. Does visual augmented feedback reduce local dynamic stability while walking? Gait Posture 2015, 42, 415-418. [CrossRef]

16. Hatzitaki, V.; Stergiou, N.; Sofianidis, G.; Kyvelidou, A. Postural Sway and Gaze Can Track the Complex Motion of a Visual Target. PLoS ONE 2015, 10, e0119828. [CrossRef] [PubMed]

17. Sotirakis, H.; Kyvelidou, A.; Mademli, L.; Stergiou, N.; Hatzitaki, V. Aging affects postural tracking of complex visual motion cues. Exp. Brain Res. 2016, 234, 2529-2540. [CrossRef] [PubMed]

18. Suzuki, Y.; Nomura, T.; Casadio, M.; Morasso, P. Intermittent control with ankle, hip, and mixed strategies during quiet standing: A theoretical proposal based on a double inverted pendulum model. J. Theor. Boil. 2012, 310, 55-79. [CrossRef] [PubMed]

19. Cofré Lizama, L.E.; Pijnappels, M.; Reeves, N.P.; Verschueren, S.M.P.; van Dieën, J.H. Frequency domain mediolateral balance assessment using a center of pressure tracking task. J. Biomech. 2013, 46, 2831-2836. [CrossRef]

20. Halliday, D. A framework for the analysis of mixed time series/point process data-Theory and application to the study of physiological tremor, single motor unit discharges and electromyograms. Prog. Biophys. Mol. Boil. 1995, 64, 237-278. [CrossRef]

21. Dingwell, J.B.; Cusumano, J.P.; Cavanagh, P.R.; Sternad, D. Local dynamic stability versus kinematic variability of continuous overground and treadmill walking. J. Biomech. Eng. 2001, 123, 27-32. [CrossRef]

22. England, S.A.; Granata, K.P. The influence of gait speed on local dynamic stability of walking. Gait Posture 2007, 25, 172-178. [CrossRef] [PubMed]

23. Ekizos, A.; Santuz, A.; Arampatzis, A. Transition from shod to barefoot alters dynamic stability during running. Gait Posture 2017, 56, 31-36. [CrossRef] [PubMed]

24. Packard, N.H.; Crutchfield, J.P.; Farmer, J.D.; Shaw, R.S. Geometry from a time series. Phys. Rev. Lett. 1980, 45, 712. [CrossRef]

25. Fraser, A.M.; Swinney, H.L. Independent coordinates for strange attractors from mutual information. Phys. Rev. A 1986, 33, 1134-1140. [CrossRef] [PubMed]

26. Ekizos, A.; Santuz, A.; Schroll, A.; Arampatzis, A. The Maximum Lyapunov Exponent during Walking and Running: Reliability Assessment of Different Marker-Sets. Front. Physiol. 2018, 9, 1-11. [CrossRef] [PubMed]

27. Rosenstein, M.T.; Collins, J.J.; De Luca, C.J. A practical method for calculating largest Lyapunov exponents from small data sets. Phys. D Nonlinear Phenom. 1993, 65, 117-134. [CrossRef]

28. Varlet, M.; Coey, C.A.; Schmidt, R.C.; Marin, L.; Bardy, B.G.; Richardson, M.J. Influence of stimulus velocity profile on rhythmic visuomotor coordination. J. Exp. Psychol. Hum. Percept. Perform. 2014, 40, 1849-1860. [CrossRef]

29. Varlet, M.; Schmidt, R.C.; Richardson, M.J. Influence of stimulus velocity profile on unintentional visuomotor entrainment depends on eye movements. Exp. Brain Res. 2017, 235, 3279-3286. [CrossRef]

30. Wolpe, N.; Wolpert, D.M.; Rowe, J.B. Seeing what you want to see: Priors for one's own actions represent exaggerated expectations of success. Front. Behav. Neurosci. 2014, 8, 1-14. [CrossRef]

31. Bays, P.M.; Wolpert, D.M. Computational principles of sensorimotor control that minimize uncertainty and variability. J. Physiol. 2007, 578, 387-396. [CrossRef] [PubMed]

32. Franklin, D.W.; Wolpert, D.M. Computational Mechanisms of Sensorimotor Control. Neuron 2011, 72, 425-442. [CrossRef] [PubMed]

33. Wu, H.G.; Miyamoto, Y.R.; Nicolas, L.; Castro, G.; Smith, M.A.; Biology, E. Temporal structure of motor variability is dynamically regulated and predicts motor learning ability. Nat. Neurosci. 2015, 17, 312-321. [CrossRef] [PubMed]

34. Herzfeld, D.J.; Shadmehr, R. Motor variability is not noise, but grist for the learning mill. Nat. Neurosci. 2014, 17, 149-150. [CrossRef] [PubMed]

35. Kennedy, M.W.; Crowell, C.R.; Villano, M.; Schmiedeler, J.P. Effects of Filtering the Center of Pressure Feedback Provided in Visually Guided Mediolateral Weight Shifting. PLoS ONE 2016, 11, e0151393. [CrossRef] [PubMed]

36. Shadmehr, R.; Smith, M.A.; Krakauer, J.W. Error Correction, Sensory Prediction, and Adaptation in Motor Control. Annu. Rev. Neurosci. 2010, 33, 89-108. [CrossRef] [PubMed] 
37. Posner, M.I.; Nissen, M.J.; Klein, R.M. Visual dominance: An information-processing account of its origins and significance. Psychol. Rev. 1976, 83, 157-171. [CrossRef] [PubMed]

38. Fitts, P.M. Cognitive aspects of information processing: III. Set for speed versus accuracy. J. Exp. Psychol. 1966, 71, 849-857. [CrossRef] [PubMed]

39. Piras, A.; Raffi, M.; Perazzolo, M.; Squatrito, S. Influence of heading perception in the control of posture. J. Electromyogr. Kinesiol. 2018, 39, 89-94. [CrossRef] [PubMed]

40. Balestrucci, P.; Daprati, E.; Lacquaniti, F.; Maffei, V. Effects of visual motion consistent or inconsistent with gravity on postural sway. Exp. Brain Res. 2017, 235, 1999-2010. [CrossRef] [PubMed]

41. Stepp, N. Anticipation in feedback-delayed manual tracking of a chaotic oscillator. Exp. Brain Res. 2009, 198, 521-525. [CrossRef] [PubMed]

42. Stephen, D.G.; Stepp, N.; Dixon, J.A.; Turvey, M. Strong anticipation: Sensitivity to long-range correlations in synchronization behavior. Phys. A Stat. Mech. Its Appl. 2008, 387, 5271-5278. [CrossRef]

43. Hajnal, A.; Richardson, M.J.; Harrison, S.J.; Schmidt, R.C. Location but not amount of stimulus occlusion influences the stability of visuo-motor coordination. Exp. Brain Res. 2009, 199, 89-93. [CrossRef] [PubMed]

44. Danion, F.; Duarte, M.; Grosjean, M. Fitts' law in human standing: The effect of scaling. Neurosci. Lett. 1999, 277, 131-133. [CrossRef]

45. Peterka, R.J. Sensorimotor Integration in Human Postural Control. J. Neurophysiol. 2002, 88, 1097-1118. [CrossRef] [PubMed]

46. Morasso, P.G.; Schieppati, M. Can Muscle Stiffness Alone Stabilize Upright Standing? J. Neurophysiol. 1999, 82, 1622-1626. [CrossRef]

(C) 2019 by the authors. Licensee MDPI, Basel, Switzerland. This article is an open access article distributed under the terms and conditions of the Creative Commons Attribution (CC BY) license (http://creativecommons.org/licenses/by/4.0/). 